\title{
THE
}

\section{Observation of Pretransitional Divergence of Shear Viscosity near a Smectic-A-Smectic-B Phase Transition}

\author{
S. Bhattacharya
}

S. V. Letcher

University of Rhode Island, sletcher@uri.edu

Follow this and additional works at: https://digitalcommons.uri.edu/phys_facpubs

Terms of Use

All rights reserved under copyright.

\section{Citation/Publisher Attribution}

Bhattacharya, S., \& Letcher, S. V. (1979). Observation of Pretransitional Divergence of Shear Viscosity near a Smectic-A-Smectic- $B$ Phase Transition. Physical Review Letters, 42(7), 458-461. doi: 10.1103/ PhysRevLett.42.458

Available at: http://dx.doi.org/10.1103/PhysRevLett.42.458

This Article is brought to you for free and open access by the Physics at DigitalCommons@URI. It has been accepted for inclusion in Physics Faculty Publications by an authorized administrator of DigitalCommons@URI. For more information, please contact digitalcommons-group@uri.edu. 
ficient of the concentration wave, and $\varphi_{n}$ is its phase factor. Inserting (2) into (1) one finds the normal reflections at $\vec{Q}=\vec{\tau}$ (the fcc reciprocallattice vector) and satellite reflections at $\vec{Q} \pm n \vec{q}$ $=\vec{\tau}$.

We have made intensity calculations for the satellite reflections assuming different deuterium-vacancy configurations on the 420 planes. A configuration of four fully occupied planes followed by one vacant, that corresponds to the $\mathrm{Ni}_{4} \mathrm{Mo}$ ordered structure, is in reasonable agreement with the data; however, a sizable displacement of the $\mathrm{D}$ atoms from the octahedral sites, involving relaxation toward the vacant planes, is indicated by the calculations. A complete description of the structure including $\mathrm{x}$-ray diffraction data will be published later.

Recently, Anderson and co-workers ${ }^{5}$ reported a peak at $\left(1, \frac{1}{2}, 0\right)$ in reciprocal space (point $W$ in Fig. 3) in a sample of $\mathrm{PdD}_{0.64^{\circ}}$ This observation is consistent with the predictions of Khachaturyan ${ }^{6}$ and Goldberg ${ }^{7}$ which include an ordering wavelength of four $(4,2,0)$ planes, or $(2 / \sqrt{5}) a$, where $a$ is the lattice constant. The wavelength derived from our results of five $(4,2,0)$ planes, or $(\sqrt{5} /$ 2) $a$, is not in agreement with the above predictions. However, according to the suggestion of Goldberg and Manchester, ${ }^{8}$ the results of Anderson and co-workers can be reconciled with the present results within the framework given by de Fontaine. ${ }^{9}$
The technical assistance of Richard Hitterman and Harold Knott is acknowledged, as is support from the National Science Foundation (Grant No. DMR77-08463), the U. S. Department of Energy, and the Argonne Center for Educational Affairs.

(a) Present address: Hughes Aircraft Co., P. O. Box 92919, Los Angeles, Calif. 90009.

'J. K. Jacobs and F. D. Manchester, J. Less Common. Metals 49, 67 (1976), and references contained therein.

${ }^{2}$ C. B. Satterthwaite, T. E. Ellis, and R. J. Miller, in Transition Metals-1977, The Institute of Physics Conference Series No. 39, edited by M. J. G. Lee, J. M. Perz, and E. Fawcett (The Institute of Physics, Bristol and London, 1978), p. 501; T. E. Ellis, thesis, University of Illinois, 1978 (unpublished).

${ }^{3}$ L. Heaton, M. H. Mueller, M. F. Adam, and R. L. Hitterman, J. Appl. Crystallogr. $\underline{3}, 289$ (1970).

${ }^{4}$ M. H. Mueller, J. Faber, Jr., H. E. Flotow, and D. G. Westlake, Acta Crystallogr. 31, 599 (1975).

${ }^{5}$ I. S. Anderson, C. J. Carlile, and D. K. Ross, J. Phys. C 11, L381 (1978); I. S. Anderson, D. K. Ross, and C. J. Carlile, Phys. Lett. 68A, 249 (1978).

${ }^{6}$ A. G. Khachaturyan, Phys. Status Solidi B $\underline{60}, 9$ (1973).

${ }^{7}$ H. A. Goldberg, in Transition Metals-1977, The Institute of Physics Conference Series No. 39, edited by M. J. G. Lee, J. M. Perz, and E. Fawcett (The Institute of Physics, Bristol and London, 1978), p. 504.

${ }^{8}$ H. A. Goldberg and F. D. Manchester, Phys. Lett. $68 \mathrm{~A}, 360$ (1978).

${ }^{9} \mathrm{D}$. de Fontaine, Acta Metall. 23, 553 (1975).

\title{
Observation of Pretransitional Divergence of Shear Viscosity near a Smectic- $A-$ Smectic- $B$ Phase Transition
}

\author{
S. Bhattacharya and S。V. Letcher \\ Department of Physics, University of Rhode Island, Kingston, Rhode Island 02881, and Francis Bitter National \\ Magnet Laboratory, Massachusetts Institute of Technology, Cambridge, Massachusetts 02139
}

(Received 12 October 1978)

\begin{abstract}
Strong pretransitional divergence of shear viscosity was observed for the first time near a smectic- $A$-smectic- $B$ phase transition by capillary shear flow with the layer normal perpendicular to both the flow velocity and velocity gradient. The results are inconsistent with a single power-law type of critical exponent and are suggestive of an essential singularity. Possible implications of the results for recently proposed models of the smectic- $B$ phase and mechanisms for smectic- $A-$ smectic- $B$ phase transitions are discussed.
\end{abstract}

Flow properties of nematic liquid crystals have undergone extensive studies in recent years. ${ }^{1}$ Pretransitional divergence of shear viscosity near a nematic-smectic- $A$ phase transition was observed by capillary flow for the director parallel to the flow velocity and perpendicular to the velocity gradient. ${ }^{2,3}$ The smectics, in comparison, have received little attention. The flow behavior near the smectic- $A$ to smectic- $B$ phase transition, in particular, has not been studied, to the best of our knowledge.

We present here results of a capillary-shear- 
flow study near a smectic-A-smectic-B (hereafter referred to as $A$ and $B$ ) phase transition. The material studied was butyloxy-benzylidene-octylaniline (BBOA, also referred to as 40.8$),{ }^{4}$ which has the following transition temperatures:

$$
\text { solid } \stackrel{37.77^{\circ} \mathrm{C}}{\longrightarrow} \text { smectic }-B \stackrel{49.2^{\circ} \mathrm{C}}{\longrightarrow} \text { smectic }-A \stackrel{63.7^{\circ} \mathrm{C}}{\longrightarrow} \text { nematic } \stackrel{78.6^{\circ} \mathrm{C}}{\longrightarrow} \text { isotropic。 }
$$

The latent heat at the $A-B$ transition is $0.5 \mathrm{kcal} /$ mole. The material was obtained from the CPAC, Inc., Organic Specialties Group and used without further purification.

Figure 1 shows the geometry of the experiment. We used a flat capillary viscometer of rectangular cross section, which consists of two flat glass plates separated by a Mylar spacer (thickness $=360 \mu \mathrm{m})$ in which a slit of length $45 \mathrm{~mm}$ and width $7 \mathrm{~mm}$ was cut. Holes in one plate at each end of the slit provide for entrance and exit of the material through glass tubes connected to the holes. Surface treatment and an applied magnetic field $(\sim 80 \mathrm{kOe})$ aligned the director parallel to the glass plates and perpendicular to the flow velocity。

In this geometry, for which the flow in both the capillary and the connecting tubes is predominantly the motion of molecules within the layers, we expect relatively little defect generation at the capillary entrance and exit. The viscometer was submerged in a circulating water bath whose temperature could be controlled to $\pm 0.05 \mathrm{~K}$. The sample was cooled from the nematic phase in the presence of the magnetic field. This produced the desired orientation in the bulk and the large field strength prevented flow-alignment effects in the smectic phase.

The details of the measurement technique are given elsewhere。 ${ }^{3}$ The viscosity was measured by recording the change of the height of the liquid column in one of the tubes as a function of time. Within the flow-rate regime studied, the flow was found to be non-Newtonian in the same way as was observed in $\mathrm{N}-p$-cyanobenzylidene- $p$ octyloxyaniline (CBOOA). ${ }^{3}$ Since the capillary flow experiments are done at significantly high and nonuniform shear rates, it is not possible to extract the low-shear-rate behavior.

Even though some ambiguity exists as to the absolute value of the viscosity at any particular temperature, it is observed nevertheless that the ratio of the effective viscosities at two temperatures at the same flow rate is insensitive to the flow rate. Thus the ratio becomes an unambiguous quantity and all measurements were done relative to the viscosity at one particular temperature. The data were reproducible to within our experimental accuracy of about $\pm 5 \%$.

Figure 2 shows the temperature dependence of the normalized viscosity relative to its value at $53.4^{\circ} \mathrm{C}$ near the $A-B$ transition. A very strong pretransitional divergence occurs, as is clearly seen from the figure. The data in Fig. 2 represent a variety of flow rates, all appropriately normalized. Very close to $T_{A B}$ the viscosity becomes enormously large and accurate measurements are not possible.

In the $B$ phase the flow becomes "blocked"; no flow is observed with the maximum available pressure $(550 \mathrm{~mm}$ of water head, corresponding to a shear stress of $200 \mathrm{dyn} / \mathrm{cm}^{2}$ at the capillary
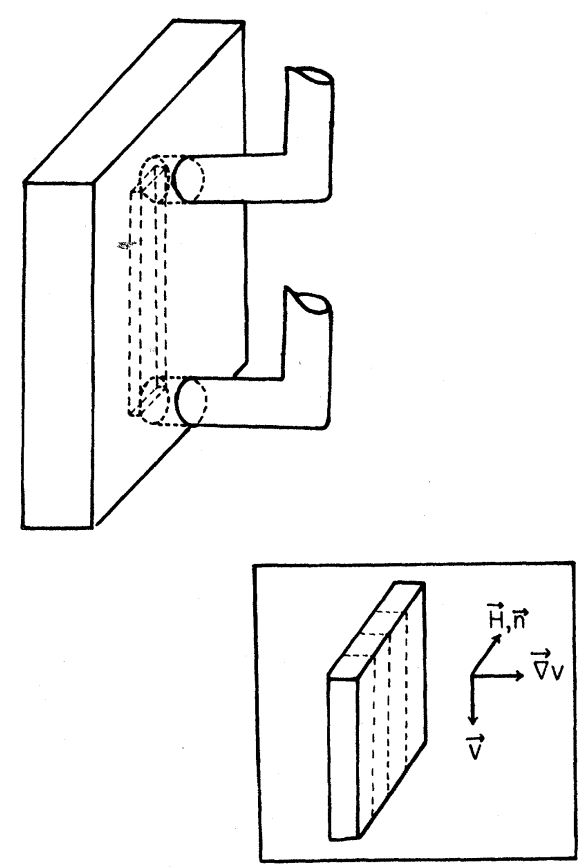

FIG. 1. Capillary cell with the entry tubes. The inset shows the geometry of flow: The normal to the smectic layers $\vec{n}$ is parallel to $\overrightarrow{\vec{H}}$. In the capillary, $\overrightarrow{\mathrm{n}}$ is perpendicular to both the flow velocity $\vec{V}$ and the velocity gradient $\nabla V$. The flow is the relative motion of molecules within a layer. In the entry tubes, the flow can be a combination of flow within the layer and the slipping of layers relative to one another, but, because of the coupling to the capillary, the former is expected to dominate. 


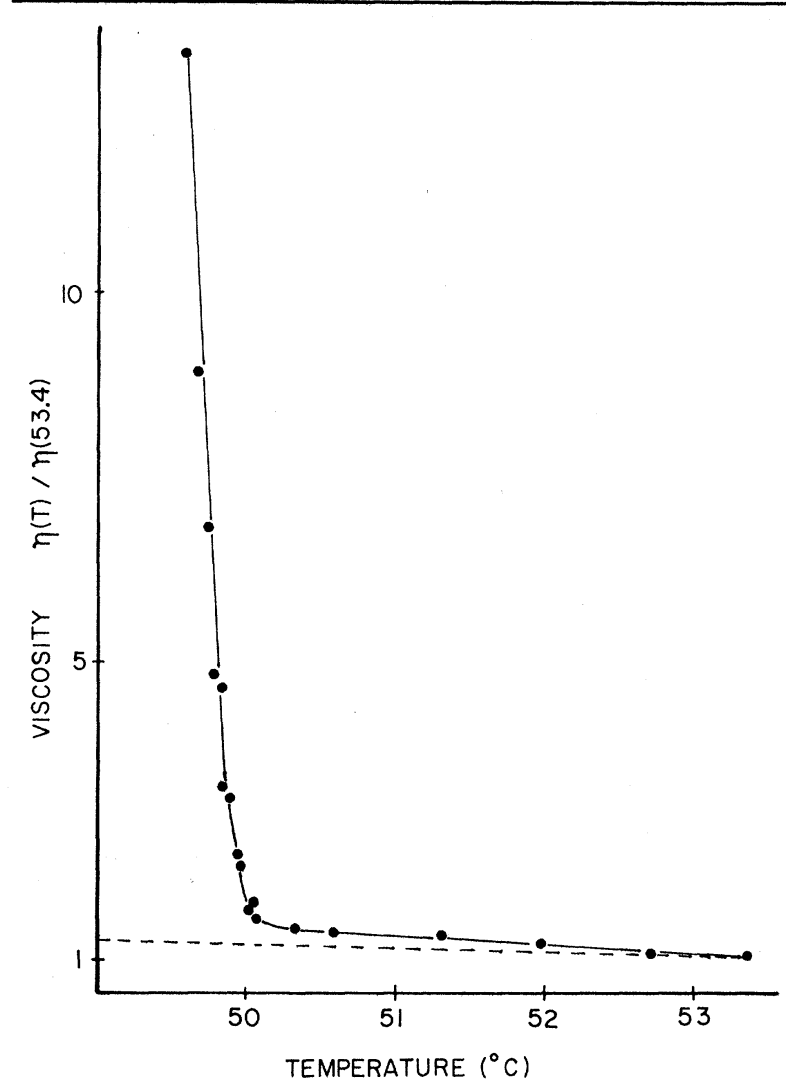

FIG. 2. Temperature dependence of the normalized viscosity near the $A-B$ phase transition. $\eta(53.4)$ represents the viscosity at $53.4^{\circ} \mathrm{C}$, which is the normalization constant. The dashed line represents the classical Arrhenius background with an activation energy of 11 $\mathrm{kcal} / \mathrm{mole}$.

walls). For this geometry, this means that molecular motion within the layers becomes frozen, but says nothing about relative motion of the layers.

Throughout the smectic $-A$ phase, from 63 to $53^{\circ} \mathrm{C}$, the viscosity exhibited an Arrhenius behavior in agreement with measurements in CBOOA in the same geometry. ${ }^{3}$ The activation energy was estimated to be $11 \mathrm{kcal} / \mathrm{mole}$, also in good agreement with the value in CBOOA. Therefore, in order to extract the critical part of the viscosity, we subtracted this noncritical Arrhenius background, represented by the dashed line in Fig. 2 。

The temperature dependence of the critical part of the normalized viscosity, i.e., $\eta_{\mathrm{cr}} /$ $\eta\left(53.4^{\circ}\right)$, obtained after subtracting the background, is shown in Fig. 3 in the form of a log$\log$ plot. The data appear to be inconsistent with a unique critical exponent. Nearer to $T_{A B}$, the

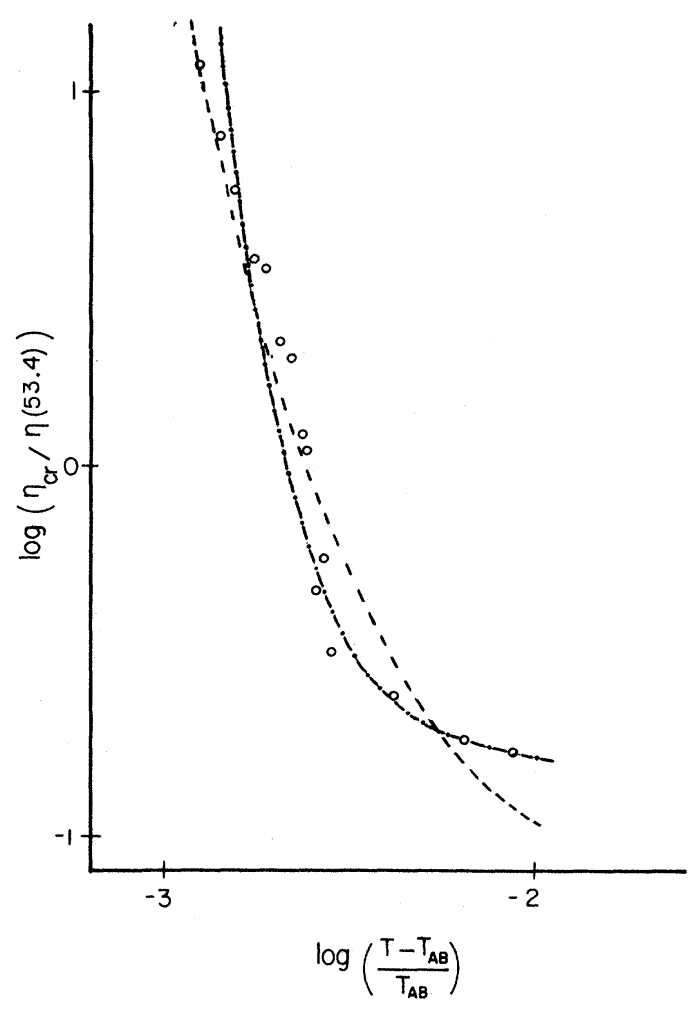

FIG. 3. Log-log plot of the critical viscosity with temperature. The dashed curves and dash-dotted curves are fits using Eq. (1) with $\eta_{0} / \eta(53.4)=0.05$ and 0.15 , respectively. The corresponding values of $n$ are 0.8 and 2.0 .

fit with a single exponent improves but the apparent exponent is very large, $4.0 \pm 0.2$ (as compared to $\sim 0.33$ near the nematic-smectic $-A$ transition)。 Farther away from $T_{A B}$, the average exponent is about $0.7 \pm 0.1$. This behavior is suggestive of an essential singularity of the form

$$
x=x_{0} \exp \left[b /\left(T-T_{c}\right)^{n}\right]
$$

where, in our case, $x$ is the shear viscosity. The problems associated with fitting such a form are quite severe. ${ }^{5}$ It is found that the value of $n$ is very sensitive to the choice of $\eta_{0}$, which is not known a priori. For example, for $\eta_{0} / \eta\left(53.4^{\circ}\right)$ $=0.05$ and 0.15 the values of $n$ are found to be 0.8 and 2.0 , respectively. It was shown by series expansion methods ${ }^{5}$ that an essential singularity of this type yields, in a standard log-log plot, a large apparent exponent. From all this we would like to emphasize that our data seem to be indicative of an essential singularity.

Unfortunately, no theoretical predictions are 
available at this time for the form of divergence of the shear viscosity near a bulk $A-B$ transition. Several models of the $B$ phase and possible mechanisms of the $A-B$ transition have been proposed. We will discuss the possible implication of our results in the light of these models. Model (1), proposed by de Gennes and Sarma, ${ }^{6}$ assumes that the $B$ phase results from a stacking of two-dimensional harmonic systems, differing from the solid in that no Bragg peaks are observed in the plane of the layer, and from the $A$ phase in that the structure factor has a sixfold symmetry in the plane. Model (2), proposed by Huberman, Lublin, and Doniach, ${ }^{7}$ and model (3), proposed by Birgenau and Litster, use the KosterlitzThouless ${ }^{9}$ dislocation-mediated picture of melting in two dimensions as the framework for the $A-B$ transition, but have different models for the $B$ phase. Model (2) assumes that the $B$ phase results from a stacking of two-dimensional solids, and unbinding of the dislocation pairs drives it into the $A$ phase. Model (3) is based on theoretical work on two-dimensional melting by Nelson and Halperin, ${ }^{10,11}$ who recently predicted the existence of a "hexatic" phase intermediate between the solid and liquid phases in two dimensions. In this phase the dislocation pairs become dissociated, but disclinations still occur in bound pairs with opposite "disclinicity。" As a result, there is short-range positional order (exponential decay of the positional order parameter) and quasilong-range bond orientational order (power-law decay of the orientational order parameter). In smectic model (3), the solid, $B$ phase, and $A$ phase are assumed to result from a three-dimensional stacking of layers of the Halperin-Nelson two-dimensional solid, hexatic, and liquid phases, respectively. As a result of the interplanar coupling in the three-dimensional system, the orientational order is expected to become truly long range in the $B$ phase, but stays short range in the $A$ phase. Thus the $A-B$ phase transition is characterized by the onset of the true long-range orientational order while the positional order remains short range for both phases.

In model (1), the lattice structure in the smectic- $B$ plane would prevent flow even though the positional order is quasi long range. The nature of the divergence is not known. In model (2), the viscosity is inversely proportional to the density of isolated dislocations. As the transition is approached from above, the dislocations start binding and the rapid decrease in the free dislocation density would result in a divergence of viscosity as the square of the correlation length, which in two dimensions diverges with an essential singularity of the form of Eq. (1), with $n=0.369 . \ldots 10$ It is not known whether the existence of long-range bond orientational order would result in a diverging viscosity in model (3). In the purely twodimensional case a divergence of the viscosity in the same fashion as in model (2), i.e., as an essential singularity, is predicted near the hexatic to solid transition ${ }^{11}$; the viscosity is expected to be finite near the liquid-hexatic phase transition. In bulk systems the interlayer coupling becomes important and changes the quasi-long-range orientational order to a true long-range order, but it is not clear what effect this would have on the viscosity of the $B$ phase or on the viscosity near the $A-B$ transition.

In conclusion, we have observed, for the first time, a pretransitional divergence of shear viscosity near an $A-B$ phase transition for flow within the smectic layers. The results are suggestive of an essential singularity. This would seem to support the contention that the smectic $A-B$ transition behaves as a suitable three-dimensional modification of a two-dimensional melting process, but whether this is the case, and if so which model is correct, awaits the development of the dynamics of the smectic models.

We thank J. C. Bonner, D。R. Nelson, J. D. Litster, and P. S. Pershan for very helpful discussions and $\mathrm{J}$. C. Bonner for a critical reading of the manuscript. This work was supported by the National Science Foundation under Grant No. DMR77-07813。

${ }^{1}$ M. Miesowicz, Nature 158, 27 (1946); Ch. Gähwiller, Mol. Cryst. Liq. Cryst. 20, 301 (1973).

${ }^{2}$ L. Leger and A. Martinet, J. Phys. (Paris), Colloq. 37, C3-89 (1976).

${ }^{3}$ M. G. Kim, S. Park, Sr., M. Cooper, and S. V. Letcher, Mol. Cryst. Liq. Cryst. 36, 143 (1976).

${ }^{4}$ G. W. Smith and Z. G. Gardlund, J. Chem. Phys. 59, 3214 (1973).

${ }^{5}$ W. J. Camp and J. P. Van Dyke, J. Phys. C $\underline{8}, 336$ (1975).

${ }^{6}$ P. G. de Gennes and G. Sarma, Phys. Lett. 38A, 219 (1972).

${ }^{7}$ B. A. Huberman, D. M. Lublin, and S. Doniach, Solid State Commun. 17, 485 (1975).

${ }^{8}$ R. J. Birgeneau and J. D. Litster, to be published.

${ }^{9}$ J. M. Kosterlitz and D. J. Thouless, J. Phys. C $\underline{6}$, 1181 (1973).

${ }^{10}$ B. I. Halperin and D. R. Nelson, Phys. Rev. Lett. $\underline{41}$, 121, 519(E) (1978).

${ }^{11}$ D. R. Nelson and B. I. Halperin, Phys. Rev. B (to be published). 